\title{
A New Measure of General Trait Neuroticism about Body Signals and Worry Specific to Body Signals: The Body Signals Attention and Worry Scale (BSAWS)
}

\author{
Dr Barbara Lopes \\ Division of Psychology \\ De Montfort University \\ Leicester, UK \\ blopes@dmu.ac.uk
}

\author{
Dr Jess Hall \\ Division of Psychology \\ De Montfort University \\ Leicester, UK \\ j.hall@dmu.ac.uk
}

\author{
Dr Hui Yu \\ Division of Psychology \\ De Montfort University \\ Leicester, UK \\ hui.yu@dmu.ac.uk
}

\begin{abstract}
A new scale was developed to measure trait neuroticism and state worry about bodily signals (the BSAWS). 72 British participants were recruited to complete a heartbeat counting task and then a battery of questionnaires comprising of the BSAWS, the state-trait anxiety inventory (STAI), the emotion regulation questionnaire (ERQ) and the paranoid checklist (PC) and state social paranoia scales (SSPS). Confirmatory factor analysis supported a four-factor model of the BSAWS, with factors of 'trait neuroticism', 'trait unconcern', 'state mindfulness' and 'state worry'. These results broadly support a two-dimensional model of bodily signals' attention and worry. Correlational analyses showed concurrent validity of the BSAWS construct with other previously established measures of anxiety, paranoia and emotional regulation. Applications for the scale include measurement of attention and worry during different tasks and/or behaviours, with the potential for clinical use to study the aetiology of various body-related mental health disorders.
\end{abstract}

Keywords-trait neuroticism, worry, bodily signals, health anxiety, interoception, state anxiety, paranoia

\section{INTRODUCTION}

Recurrent, negative thoughts are an important feature of anxiety disorders. These recurrent and persistent negative thoughts are usually defined as worry. Worry can be described as an apprehensive expectation of possible negative outcomes in future events [1]. Worry is also conceptualized as an effective short-term response to uncertainty that can become self-perpetuating and anxiety maintaining with adverse longterm consequences [2]. Worry reduces subjective uncertainty, contributes to a sense of vigilance and preparedness, dampens autonomic arousal, and fuels the belief that uncertain events and overall risk can be controlled [2]. When such relief is coupled with the likely non-occurrence of low-probability feared events (in this case, a problematic health condition, e.g. arrhythmia), it can powerfully reinforce the worry response, shaping beliefs that worry is adaptive and can prevent bad things from happening. Worry is also a form of emotional suppression and cognitive avoidance that becomes selfperpetuating, in part because it blocks other emotions such as fear or anger [2] and provides an illusion of control.

\section{Trait Neuroticism and Worry: General and Specific to Bodily Signals}

There is evidence in the literature to suggest that worry is a manifestation of the dispositional trait of neuroticism, believed to reflect the general predisposition to develop psychopathological symptoms such as anxiety and depression [3]. The personality trait of neuroticism refers to relatively stable tendencies to respond with negative emotions to perceived threat, frustration, or loss. Individuals in the population vary markedly on this trait. Some individuals may exhibit frequent and intense emotional reactions to minor challenges, whereas others may respond with little emotional reaction even in the face of significant difficulties [4]. Individuals who score high on neuroticism respond more poorly to stressors, are more likely to interpret ordinary situations and stimuli as threatening, minor frustrations as hopelessly difficult and are more prone as well to develop anxiety and depressive disorders [5]. Unsurprisingly then, there is accumulating evidence demonstrating that worry is indeed linked to neuroticism [6,7]. More recently, a study has found that worry mediates the relationship between neuroticism and anxiety, meaning that worry is a cognitive coping mechanism 
that is typical of neurotic traits and may act as a way of anticipating and controlling for threat, both internal (e.g. body signals) and external (e.g. social threat, such as criticisms) [8].

Cognitive models of health anxiety [2,9,10] have long proposed that certain individuals have a persistent concern about their health. Within these models, individuals experiencing this form of anxiety, worry about the possibility of having a serious health condition because they have an enduring tendency to misinterpret bodily variations and body sensations or any other health related information. When worry is specific to one's health and this is persistent and severe then clinically speaking this is referred to as hypochondriasis $[10,11]$. Research has established that links exist between neuroticism and hypochondriasis and health anxiety [12]. For example a study has found that $29 \%$ of hypochondriacal concerns in a non-clinical population were accounted for by neuroticism [12]. This means that neuroticism may act as a predisposition trait factor for both health anxiety and hypochondriasis.

Health anxiety is conceptualised as lying in a continuum. On the one hand individuals may possess relatively normal, non-clinical concerns about their own health, and on the other hand others may possess a severe and persistent worry about their own health without evidence to support these exacerbated concerns [13]. Concerning the measurement of health anxiety, a commonly-used measure is the Health Anxiety Inventory (HAI) [13]. According to the authors the HAI is one of the few self-report measures capable of discriminating between individuals that meet the clinical criteria for hypochondriasis, and those for whom health anxiety is present but not severe, such that they do not meet the clinical criteria for hypochondriasis. The Health Anxiety Inventory has been tested in clinical populations in three studies, and the authors reported excellent psychometric properties for this measure. Moreover, the state version of the HAI was also found to be sensitive to treatment effects and able to detect changes in thinking over time [13].

In this article, the authors describe the development of a new measure of general trait neuroticism and specific worry about body signals that draws conceptually from the Health Anxiety Inventory [13]. This measure is novel in two key ways. Firstly, although the literature on health anxiety has been fairly prolific at developing measures of general health concerns (e.g. the Illness Attitudes Scale) [13,14], there are a lack of measures flexible enough to be used across different contexts to measure persistent worries and concerns about a range of different body signals (such as changes in heart rate or respiration) or activities (for example eating or exercise). Such a scale has the potential for great utility within the study of the aetiology of health anxiety and hypochondriasis. Cognitive theories of anxiety have established that anxious thoughts and worry about body signals derive from errors of thinking such as catastrophizing or making erroneous inferences without sufficient evidence to do so [15]. These errors of thinking appear to facilitate an anxious schema that can act as a lens to magnify threat (both internal and external). This maladaptive schema may then in turn contribute to anxious and threatening interpretations of internal stimuli. Incorporating measurements of worry about bodily signals in specific contexts or during specific tasks, alongside trait measures, can therefore help shed light on how moment-to-moment experience, relates to longstanding dispositions to think and feel in certain ways.

In addition, this measure will be novel in that it will move away from the general and state health anxiety constructs. It will instead adopt a circumplex model similar to circumplex models of affect [16]. This will be a two dimensional model of general trait tendencies and specific concerns exploring specifically the general negative concerns about body signals and tendency to be neurotic about one's body signals and the negative thoughts or concerns that one is reporting in regards to a specific task, activity or body signal (in this case worry about heartbeat sensation), see Fig. 1. A bidimensional model with two dimensions: context (trait vs. state) and valence (positivity vs. negativity) is proposed as basis for the new measure of trait and neurotic concern about bodily signals and worry about specific bodily signals because there is an established link between neuroticism, worry and hypochondriasis [12]. In contrast to this, mindfulness, as the state non-judgemental awareness and acceptance of the body experiences [17] has been found to be inversely related with worry, neuroticism and negative affect $[18,19]$. As such it is expected that this new measure should produce positively and strongly related dimensions of trait neuroticism and worry about specific bodily signals.

\section{Worry and Emotional Regulation of Body Experiences}

It is proposed that a measure that taps into general and specific concerns about physical sensations should draw from previous research that looks not only at the links between neuroticism and worry [8] but also at the links between neuroticism, affect related emotional regulation and stress [20].

Emotional regulation is a concept involving a set of processes that allow the individual to influence the emotions that they are experiencing, when they occur and how to understand and express these emotions [21]. Individuals often increase, maintain and decrease both negative and positive emotions [22].

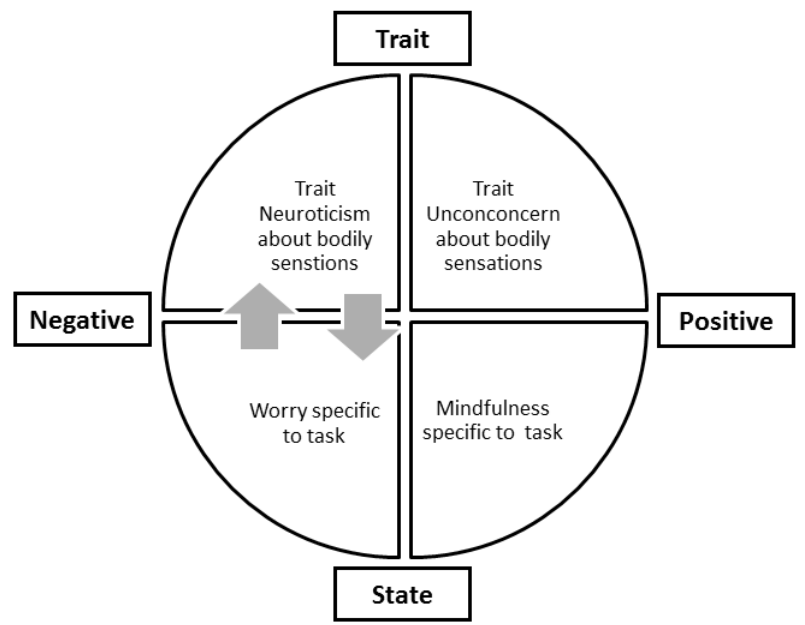

Figure 1. Circumplex Model of the Body Signals and Attention Worry Subscales 
There are many cases where emotional regulation occurs in a conscious way, for example changing a topic when it becomes upsetting, however emotional regulation may occur unconsciously, for example when one immediately moves attention away from something upsetting [22]. Another important element of emotional regulation is that fundamentally, it is neither good nor bad [23].

Researchers [22,23] have established two common emotional regulation strategies: emotional reappraisal and suppression. These strategies aim to either decrease, or increase emotional response tendencies or affective states [21]. Cognitive reappraisal is a type of cognitive change, and thus antecedent focused. Reappraisal helps the individual to reinterpret emotional stimuli, in an unemotional way [21]. Suppression on the other hand, acts as a prevention of unwanted emotional processes, during a state of emotional arousal by reducing emotion-expressive behaviour [21] for example such as the act of 'holding a poker face' when gambling. Research has demonstrated that both the suppression and reappraisal of emotional stimuli reduces negative affect. Both strategies have been shown to be effective, as brain areas that are associated with emotional processing were highly activated during the use of suppression strategies [21]. Furthermore, researchers observed physiological responses that were also present when the emotional networks were highly active, such as that skin conductance was greater, indicating that emotional regulation is effective for both physical and psychological components [24].

There is research to suggest that individuals higher in neuroticism are more likely to experience worry in cognitively demanding situations, to improve performance and to have impaired cognitive reappraisal by demonstrating a trait congruent and emotionally charged appraisal of situations, which means perceiving situations in a negative and emotionally charged way [20]. Research has also revealed that cognitive reappraisal can replace negative stress with positive stress. Some studies have suggested that individuals who selfreport greater use of reappraisal in their day to day life may experience more adaptive social and emotional outcomes [25]. Studies conducted through field, laboratory and clinical settings have shown that cognitive reappraisal is a highly effective emotional regulation technique and has been linked with positive affect [25].

This literature thus suggests that cognitive reappraisal is associated with less negative affect and worry and on the other hand affect-based emotion regulation strategies are associated not only to neuroticism but to more negative affect and worry. As such, it is expected that in this new measure of trait neuroticism about bodily signals and worry specific to a body signal, the scores of worry specific to a body signal as well as trait neuroticism about body signals will both show a positive relationship with state negative affect.

\section{Worry and Non-clinical Paranoia and Anxiety}

Paranoia (unfounded ideas of deliberate harm from others) is a key component of psychotic experience. Recent research in paranoia has advocated for a continuum between experiences of paranoia that are built upon common interpersonal concerns (such as being accepted by others or being at risk of being ostracised and harmed by others) at one end of the continuum and the development of psychotic persecutory delusions at the severe end of the continuum [26]. Cognitive models of paranoia [26] have long proposed that paranoia may be related to anomalous perception of both internal and external signals and stimuli and that non-clinical paranoia shares commonalities with non-clinical anxiety. Both are related to an anticipation of threat or danger and both are associated with worry since worry is characterised by constant intrusive thoughts about possible dangers [27]. Indeed, studies with a non-clinical population have found that anxiety, worry, and depression predict the occurrence of non-clinical persecutory ideation in an experimental situation [27, 28]. Moreover a longitudinal study with the general population was able to establish that insomnia, worry, anxiety and depression were strong predictors both of the development and the persistence of paranoid thinking [27].

Since non-clinical paranoia shares commonalities with anxiety and is associated with worry, it is expected that when we measure worry about specific body signals, this will be significantly associated with both non-clinical paranoid and anxious thoughts. This is because individuals in the general population that report non-clinical paranoid thoughts have a tendency to worry about body sensations and usually report atypical experiences that have to do with a sense that 'things don't seem and don't feel right', i.e. a sense of anomaly, which leads to constant worry about their physical and mental wellbeing [28].

\section{Aims of Study}

1. To develop a scale to measure context dependent (trait versus state) and valenced (positivity versus negativity) appraisals of bodily signals.

2. Develop sub-scales that will measure worry and mindfulness during a specific task, in this instance, a heartbeat-counting task.

\section{METHOD}

\section{Participants}

72 participants (58 female and 13 male) were recruited from the De Montfort University undergraduate population. Participants were rewarded course credit in return for participation. The mean age of participants was 21.63 years $(S D=6.16$ years $)$. One participant declined to report their age and gender. Participants were asked if they had been previously diagnosed with a psychiatric condition. 5 participants responded that they had. 21 participants declined to state whether they had or had not. There were no statistically significant differences between the participants that reported being diagnosed with a psychiatric condition and the participants that did not report having a psychiatric condition for the BSAWS and the other psychological measures. 


\section{Instruments}

\section{State-Trait Anxiety Inventory (STAI) [29]}

Participants were asked how they currently felt by using the items in the State-Trait Anxiety Inventory (20 items). Positive affect was measured by 10 items that evaluate current feeling of positive emotions (e.g. I am relaxed), whereas negative affect was measured by 10 items that evaluate current feeling of negative emotions (e.g. I feel nervous). Each item is rated on a 4 point scale ( $1=$ Not at all, $4=$ Very much so). The reliability was high for both subscales in the current study, with Cronbach's $\alpha=.92$ for positive affect and Cronbach's $\alpha=.90$ for negative affect. The score of each subscale was calculated by totalling the ratings of positive or negative feelings, with the range being 10 to 40 for each subscale. A higher score indicated stronger state feeling of positive or negative emotions respectively.

\section{The Body Signals Attention and Worry Scale (BSAWS)}

The Body Signals Attention and Worry Scale (BSAWS) was devised with the intent to measure on the one hand trait health concerns and worry about body signals and on the other hand to measure state personal experiences of body cues during a specific task or behaviour, in this instance a heartbeatcounting task. Participants were asked to rate on a 7-point Likert scale (ranging from $1=$ strongly disagree to $7=$ strongly agree) how much they agreed with 14 statements, measuring four factors. The four factors were adopted to support a circumplex models of affect, that considers emotions as valenced and both context-dependent and trait-determined. The first factor, trait neuroticism about bodily signals, was composed of 3 items that measured the general worry associated with bodily signals. One example item is "If I feel any discomfort I start to worry that something might be wrong". A higher score indicates trait tendency to be hypochondriac. The second factor, trait unconcern about bodily signals, was composed of 4 items, which measured the behaviour of deemphasising the importance of bodily signals. One example item is "If I have a bodily sensation I rarely wonder what it means". A higher score on this dimension indicates a lesser tendency to worry about bodily signals. The third factor, state worry (about heartbeat signals), was composed of 4 items that measure anxiety to specific bodily sensations in the moment. One example item is "When I was counting my heartbeats all sorts of negative thoughts went through my mind". A higher score on this subscale indicates more distress associated with performing the heartbeat counting task. The fourth factor, state mindfulness (to heartbeat signals), was composed of 3 items, which measured the enhanced calm associated with observing internally the bodily signal during the (heartbeat counting) task. One example item is "Listening to my heart enabled me to focus on the moment". A higher score on this subscale indicates better concentration and focus associated with feeling bodily signals.

\section{Emotional Regulation Questionnaire [30]}

The Emotion Regulation Questionnaire is an established 10 -item self-report questionnaire measuring two commonly used strategies, cognitive reappraisal and expressive suppression. The cognitive reappraisal subscale was used in the current study. Participants were again asked to respond on a 7point Likert scale, (ranging from $1=$ strongly disagree to $7=$ strongly agree). The cognitive reappraisal subscale is comprised of 6 items and one example is "I control my emotions by changing the way I think about the situation I'm in". A total score was calculated by summing the ratings of the six items (ranged 6-42), and a higher score indicates that cognitive reappraisal is more habitually used. The scale has been widely used in research and the internal reliability of the scale was high in the current study with Cronbach's $\alpha=.89$.

\section{Paranoia Checklist (PC) [31]}

The PC is an 18-item self-report multidimensional scale developed to measure paranoid ideation. Items range from more common thoughts about interpersonal concerns e.g. "There might be negative comments being circulated about me" and the possibility of a threat posed by others e.g. "I need to be on my guard against others" to delusional thoughts of thought control e.g. "I can detect coded messages about me in the press/TV/radio" and of conspiracy e.g. "There is a possibility of a conspiracy against me". Each item is rated on 5-point Likert scales for frequency, degree of conviction, and distress and the scale has excellent internal consistency (Cronbach's $\alpha>0.90$ ) and good convergent validity. The original PC was found to have the following Cronbach's alphas: 0.89 (frequency), 0.95 (conviction) and 0.95 (distress). The measure when used within this study also showed excellent reliability with Cronbach's alphas of .94 (frequency), .93 (conviction) and .96 (distress).

\section{State Social Paranoia Scale (SSPS) [32]}

This scale measures state social paranoia. The scale has ten persecutory items (e.g. 'Someone stared at me in order to upset me'; 'Someone was trying to isolate me'; 'Someone was trying to make me distressed'), each rated on a 5-point scale. The items conform to a recent definition of persecutory ideation. Higher scores on the scale indicate greater levels of persecutory thinking. The scale has been regarded to provide excellent internal reliability, adequate test-retest reliability, convergent validity with both independent interviewer ratings and self-report measures, and divergent validity with regard to measures of positive and neutral thinking. Previous research indicated a high internal reliability for the questionnaire (Cronbach's $\alpha=0.90$ ) [32]. The Cronbach's $\alpha$ in this study for the scale was .80 .

\section{Procedure}

Testing took place in the research cubicles at De Montfort University, under controlled conditions. Informed consent was provided by all participants, who were reminded of their rights 
to withdraw, and to anonymity and confidentiality of their data. Participants undertook a short task lasting less than five minutes, in which they were asked to sit quietly and count their heartbeats. Participants then completed the BSAWS, followed by the other self-report scales, by hand. All participants were then debriefed and thanked for their time.

\section{RESULTS}

Model evaluations were examined by Chi Square Statistics and accompanying significance tests. The Confirmatory Factor Analysis Model of the BSAWS showed a $\chi^{2}(\mathrm{df}=72)=94.14$, $p=0.041$. Goodness of fit indices reported are the Root Mean Square of Approximation (RMSEA) and the Comparative Fitness Index (CFI). The model was considered to fit the data well with a CFI of .92 and RSMEA of .066. The standardized and unstandardized loadings of the four factor model of the BSAWS are summarised in Table 1.

\section{Reliability}

Reliability was established by examining the Cronbach's $\alpha$ of the four factors of the model of the BSAWS. The State Worry factor showed a Cronbach's $\alpha$ of .77 with 4 items; the State Mindfulness factor showed a Cronbach's $\alpha$ of .76 with 3 items; the Trait Neuroticism factor showed a Cronbach's $\alpha$ of . 75 with 3 items and finally the Trait Unconcern showed a Cronbach's $\alpha$ of .74 with 4 items. The Trait Neuroticism correlated significantly with state worry $(r=.48, p<.001)$, which supports previous literature examining the association between trait neuroticism and state worry [6].

\section{Validity}

Concurrent validity was established by looking at correlations between the four factors of the BSAWS with state negative affect and state positive affect, the emotional reappraisal and the dimensions of paranoid ideation (frequency, conviction and distress) and state social paranoia. As expected State Negative Emotion was associated with State Worry $(r=.41, p$ $<.001)$ and Trait Neuroticism $(r=.39, p=.001)$ of the BSAWS. Moreover, positive correlations were observed between Trait Unconcern and State Mindfulness of the BSAWS with Cognitive Reappraisal $(r=.29, p=.037 ; r=.39$, $p=.005)$. Finally, Trait Neuroticism and State Worry showed strong positive correlations with both trait and state paranoia (see Table 2).

\section{DISCUSSION}

The items of the BSAWS scale loaded on to a four factor model with factors of 'Trait Neuroticism', 'Trait Unconcern', 'State Mindfulness' and 'State Worry'. These results broadly support a two-dimensional model of bodily signals attention and worry, with dimensions of context (i.e. trait versus state) and valence (i.e. positivity versus negativity). These dimensions are in accord with circumplex models of affect which similarly consider emotions as valenced and both context-dependent and trait-determined [16]. In addition, correlational analyses showed concurrent validity of the BSAWS construct with other previously established measures of anxiety, paranoia and emotional regulation.

The BSAWS scale makes a novel contribution to the literature as it permits an understanding of individual experiences and responses during specific tasks. The scale could therefore be used for example to explore people's experiences during a range of very different tasks and behaviours, such as eating behaviours, or during physical tasks, as well as interoceptive tasks similar to the one used within the present study.

TABLE I. UNSTANDARDISED LOADINGS, STANDARD ERRORS AND STANDARDISED LOADINGS FOR 4-FACTOR CONFIRMATORY MODEL OF BSAWS

\begin{tabular}{|c|c|c|c|c|c|}
\hline Factors & Items & $\begin{array}{l}\text { Unstandardised } \\
\text { Loading }\end{array}$ & $\begin{array}{l}\text { Standard } \\
\text { Error }\end{array}$ & $\begin{array}{l}\text { Standardised } \\
\text { Loading }\end{array}$ & $p$ \\
\hline \multirow{3}{*}{$\begin{array}{c}\text { Trait } \\
\text { Neuroticism } \\
\text { about Bodily } \\
\text { Signals }\end{array}$} & When I feel physical discomfort or pain, I become upset & 1.00 & -- & 0.61 & -- \\
\hline & If I feel any discomfort I start to worry that something might be wrong & 1.21 & 0.21 & 0.82 & $* * *$ \\
\hline & $\begin{array}{l}\text { If I notice an unexplained bodily sensation I always find it difficult to } \\
\text { think of other things }\end{array}$ & 1.22 & 0.22 & 0.81 & $* * *$ \\
\hline \multirow{4}{*}{$\begin{array}{c}\text { Trait } \\
\text { Unconcern } \\
\text { about Bodily } \\
\text { Signals }\end{array}$} & I do not notice physical tension or discomfort until it becomes severe & 1.00 & -- & 0.61 & -- \\
\hline & I am not afraid of serious illness & 1.07 & 0.27 & 0.68 & $* * *$ \\
\hline & I can notice an unpleasant bodily sensation without worrying about it & 0.99 & 0.26 & 0.62 & $* * *$ \\
\hline & If I have a bodily sensation I rarely wonder what it means & 0.96 & 0.24 & 0.67 & $* * *$ \\
\hline \multirow{3}{*}{$\begin{array}{c}\text { State Worry } \\
\text { about } \\
\text { Heartbeat } \\
\text { Signal }\end{array}$} & I felt that something was wrong when counting my heartbeat & 1.00 & -- & 0.75 & -- \\
\hline & $\begin{array}{c}\text { When I was counting my heartbeats all sorts of negative thoughts went } \\
\text { through my mind }\end{array}$ & 0.84 & 0.15 & 0.70 & $* * *$ \\
\hline & I felt distressed and confused when counting my heartbeat & 0.68 & 0.12 & 0.71 & $* * *$ \\
\hline \multirow{3}{*}{$\begin{array}{c}\text { State } \\
\text { Mindfulness } \\
\text { about } \\
\text { Heartbeat } \\
\text { Signal }\end{array}$} & I felt relaxed and calm when counting my heartbeat & 1.00 & - & 0.72 & -- \\
\hline & Listening to my heart enabled me to focus on the moment & 0.90 & 0.20 & 0.66 & $* * *$ \\
\hline & When counting my heartbeats I felt I was in tune with my body & 0.97 & 0.22 & 0.76 & $* * *$ \\
\hline
\end{tabular}




\begin{tabular}{|c|c|c|c|c|c|c|c|c|c|c|c|c|c|}
\hline & \multirow[b]{2}{*}{ Mean } & \multirow[b]{2}{*}{$S D$} & \multicolumn{10}{|c|}{ Bivariate correlations } \\
\hline & & & & \multirow[b]{2}{*}{--} & \multirow[t]{2}{*}{2} & \multirow[t]{2}{*}{3} & \multirow[t]{2}{*}{4} & \multirow[t]{2}{*}{5} & \multirow[t]{2}{*}{6} & \multirow[t]{2}{*}{7} & \multirow[t]{2}{*}{8} & \multirow[t]{2}{*}{9} & \multirow[t]{2}{*}{10} \\
\hline 1 & Trait Neuroticism & 12.19 & 4.13 & & & & & & & & & & \\
\hline 2 & Trait Unconcern & 18.47 & 5.10 & .19 & -- & & & & & & & & \\
\hline 3 & State Worry & 8.56 & 4.20 & $.48^{* *}$ & -.11 & -- & & & & & & & \\
\hline 4 & State Mindfulness & 9.69 & 4.05 & .13 & .12 & .22 & -- & & & & & & \\
\hline 5 & Positive Affect & 22.47 & 7.14 & .12 & -.10 & .14 & $.39^{* *}$ & -- & & & & & \\
\hline 6 & Negative Affect & 15.50 & 6.16 & $.39^{* *}$ & -.17 & $.41^{* * * *}$ & .09 & $-.72^{* * *}$ & -- & & & & \\
\hline 7 & Cognitive Reappraisal & 30.23 & 6.69 & -.25 & $-.29^{*}$ & -.24 & $-.39^{* *}$ & $-.31^{* *}$ & -.08 & -- & & & \\
\hline 8 & State Paranoia & 15.06 & 8.12 & $.31^{* *}$ & -.14 & $.57^{* *}$ & .06 & .16 & .53 & -.24 & -- & & \\
\hline 9 & Paranoia Frequency & 31.34 & 14.01 & $.25^{*}$ & -.06 & $.53^{* * *}$ & .09 & $.29^{*}$ & .50 & -.23 & $.71^{* * *}$ & -- & \\
\hline 10 & Paranoia Conviction & 38.26 & 19.73 & .13 & .15 & $.36^{* *}$ & .10 & .21 & .37 & $-.44^{* *}$ & $.49^{* * *}$ & $.68^{* * *}$ & -- \\
\hline 11 & Paranoia Distress & 22.27 & 17.64 & $-.25^{*}$ & -.02 & $.53^{* * *}$ & .13 & $.29^{*}$ & .43 & -.24 & $.50^{* * *}$ & $.60^{* * * *}$ & $.46^{* * *}$ \\
\hline
\end{tabular}

Most similar scales have considered the relationship between concern and worry with bodily sensations and anxiety alone, however this study also considers the relationship with paranoid cognitions, i.e. how 'threatening' they experience external phenomena to be - a sense of lack of 'rightness' to situations in general. The current study's findings support previous research linking worry, trait neuroticism and trait paranoia $[27,33]$.

This measure has potential clinical implications. It could be used to look at the aetiology of health anxiety and also paranoid cognitions, by examining how individuals experience specific situations on a state-by-state basis and relating this experience to trait dimensions.

Future validation of this measure would benefit from increased sample sizes as well as sampling within clinical populations such as individuals with hypochondriac disorders, individuals with generalised anxiety disorder and individuals with both paranoid and/or somatic delusional disorders.

\section{ACKNOWLEDGMENT}

The authors would like to acknowledge Laura Allen, Nathan Spicer, Georgia Smith and Christopher Drayton for their help with data collection.

\section{REFERENCES}

[1] Borkoveck, T.D.; Robinson, E.; Pruzinsky, T. \& Depree, A. (1983) Preliminary exploration of worry: some characteristics and processes.

[2] Barlow DH. (2002) Anxiety and its disorders: the nature and treatment of anxiety and panic. 2nd ed. New York: Guilford Press.

[3] Segerstrom, S.; Jennie, C. I.; Tsao, L. \& Alden, M. (2000) Worry and rumination: Repetitive thought as a concomitant and predictor of negative mood. Cognitive Therapy \& Research, 24 (6), 671-688.
[4] Thompson, E.R. (October 2008). Development and validation of an International English Big-Five Mini-Markers. Personality and Individual Differences 45 (6): 542-548.doi:10.1016/j.paid.2008.06.01

[5] Ormel J.; Jeronimus, B.F.; Kotov, M.; Riese, H.; Bos, E.H.; Hankin, B. (2013). "Neuroticism and common mental disorders: Meaning and utility of a complex relationship". Clinical Psychology Review 33 (5): 686-697. doi:10.1016/j.cpr.2013.04.003.

[6] Davey, G. \& Tallis, F. (1994) Worrying: Perspectives on theory, assessment and treatment. Clinical Psychology Review, 15 (3), 258-259.

[7] Keogh, E., French, C.C., \& Reidy, J. (1998). Predictors of worry. Anxiety, Stress \& Coping, 11, 67-80.

[8] Muris, P.; Roelofs,J.; Rassin, E.; Franken, I. \& Mayer, B. (2005) Mediating effects of rumination and worry on the links between neuroticism, anxiety and depression. Personality and Individual Differences, 39, 1105-1111.

[9] Warwick, H., \& Salkovskis, P. (1990). Hypochondriasis. Behaviour Research and Therapy, 28, 105-117.

[10] Salkovskis, P. M. \& Bass, C. (1997). Hypochondriasis. In The Science and Practice of Cognitive-Behaviour Therapy (ed. D. M.Clark and C. G. Fairburn), pp. 313-339. Oxford University Press Oxford.

[11] American Psychiatric Association. (2013). Diagnostic and statistical manual of mental disorders, (DSM-5®). American Psychiatric Pub.

[12] Noyes, R. Jr; Stuart, S. P.; Langbehn, D. R.; Happel, R. L.; Longley, S. L.; Muller, B.A. \& Yagla, S. J. (2003) Test of an interpersonal model of Hypochondriasis. Psychological Medicine, 65 (2), 292-300.

[13] Salkovskis, P. M., Rimes, K. A., Warwick, H. M., \& Clark, D. M. (2002). The health anxiety inventory: Development and validation of scales for the measurement of health anxiety and hypochondriasis. Psychological Medicine, 32, 843-853.

[14] Kellner, R., Abbott, P., Winslow, W. W. \& Pathak, D. (1987). Fears, beliefs, and attitudes in DSM-III hypochondriasis. Journal of Nervous and Mental Diseases, 175, 20-25.

[15] Clark, D. M. \& Wells, A. (1995). A cognitive model of social phobia. In R. Heimberg, M. Liebowitz, D. A. Hope, \& F. R. Schneier (Eds.), Social phobia: Diagnosis, assessment and treatment. (pp. 69-93). New York: Guilford Press.

[16] Posner, J.; Russel, J. \& Peterson, B. (2005) The circumplex model of affect: an integrative approach to affective neuroscience, cognitive development and psychopathology. Deviant Psychopathology. 17(3), $715-734$. 
[17] Kabat-Zinn, J. (1994). Wherever you go, there you are: Mindfulness meditation in everyday life. New York:Hyperion

[18] Feltman, R.; Robinson, M. \& Ode, S. (2009) Mindfulness as a moderator of neuroticism-outcome relations: a self-regulation perspective. Journal of Research in Personality Psychology, 43, 953961.

[19] Fisak, B \& Von Leke, A. (2012) The relationship between the Five Facets of Mindfulness and worry in a non-clinical sample. Mindfulness, 3, 15-21

[20] Tamir, M. (2005) Don't worry, be happy? Neuroticism, trait consistent affect regulation and task performance. Journal of Personality and Social Psychology, 89 (3), 449-461.

[21] van der Meer, L., van't Wout, M., \& Aleman, A. (2009). Emotion regulation strategies in patients with schizophrenia. Psychiatry research, 170(2), 108-113.

[22] Gross, J. J. (2002). Emotion regulation: Affective, cognitive, and social consequences. Psychophysiology, 39(3), 281-291.

[23] Gross, J.J. (2008) Emotion regulation. Handbook of Emotions, 3, 497513

[24] Goldin, P. R., McRae, K., Ramel, W., \& Gross, J. J. (2008). The neural bases of emotion regulation: reappraisal and suppression of negative emotion. Biological psychiatry, 63(6), 577-586.

[25] Denny, B. T., \& Ochsner, K. N. (2014) .Behavioural effects of longitudinal training in cognitive reappraisal. Emotion, 14(2), 425-433.
[26] Freeman, D. \& Freeman, J. (2008) Paranoia: The $21^{\text {st }}$ century fear. Oxford: Oxford University Press.

[27] Freeman, D.; Stahl, D.; McManus, S.; Meltzer, H.; Brugha, T.; Wiles, N. \& Bebbington, P. (2012) Insomnia, worry, anxiety and depression as predictors of the occurrence and persistence of paranoid thinking. Social Psychiatry and Psychiatric Epidemiology, 47 (8), 1195-203. doi: 10.1007/s00127-011-0433-1

[28] Freeman, D.; Pugh, K. Antley, A.; Slater, M.; Bebbington, P.; Gittins, M.; Dunn, G.; Kuipers, E.; Fowler, D. \& Garety P. (2008) Virtual reality study of paranoid thinking in the general population. British Journal of Psychiatry, 192 (4), 258-263. DOI: 10.1192/bjp.bp.107.044677

[29] Spielberger, C. D., Gorsuch, R. L., Lushene, R., Vagg, P. R., \& Jacobs, G. A. (1983). Manual for the State-Trait Anxiety Inventory. Palo Alto, CA: Consulting Psychologists Press.

[30] Gross, J.J. \& John, O.P. (2003) Individual differences in two emotional regulation processes: implications for the affect, relationships, and wellbeing. Journal of Personality and Social Psychology, 85, 348-362.

[31] Freeman, D., Garety, P.A., Bebbington, P.E., Smith, B., Rollinson, R., Fowler, D., Kuipers, E., Katarzyna, R. \& Dunn, G. (2005). Psychological investigation of the structure of paranoia in a non-clinical population. British Journal of Psychiatry, 186, 427-435

[32] Freeman, D., Pugh, K., Green, C., Valmaggia, L., Dunn, G., \& Garety, P. (2007). A measure of state persecution in experimental studies. Journal of Nervous and Mental Disease, 195(9), 781-4. 